\title{
ROBUST CONTROL AND STATE ESTIMATION OF A THREE-STAGE ANAEROBIC DIGESTION PROCESS
}

\author{
Samia Semcheddine, Hanane Bouchareb
}

\begin{abstract}
The production of biogas via an anaerobic fermentation process is very interesting for various reasons: it not only produces renewable energy, but also facilitates the disposal of organic waste, therefore it preserves the environment, it contributes to the resolution of energy problems in agriculture and agro industry, it's available for everyone because the waste is available everywhere. This process takes place in continuously stirred tank bioreactor and is described by a highly nonlinear model whose parameters are often uncertain thereby requiring robust control in any process performance enhancement procedure to optimize the biogas production. Thus a robust control technique, synergetic, is proposed to improve the settling time in a fermentation based biogas production in a simulation study; the simulation study is carried out on biotechnological process described by a five-order continuous-time reaction scheme described by three stages. An extended Luenberger observer is proposed under synergetic control to observe the different concentrations because of the high-cost and unavailable measurement instrumentation. The interest of paper lies in controlling the amount of bio methane produced and the observation of all the states of the system ( 5 different populations of the complex community of bacteria and substrate concentrations acting during anaerobic digestion (AD) processes) by simply measuring the biogas produced. The monitoring, controller and observer performance are evaluated via numerical simulations showing excellent responses under the influence of control input.
\end{abstract}

Key words: Bio methane, anaerobic digestion, synergetic control, extended Luenberger observer, state estimation.

\section{INTRODUCTION}

Anaerobic digestion is a widely used biotechnological process and a promising method for solving energy and environmental problems in agriculture and agro industry. In such types of processes, usually carried out in continuous stirred tank bioreactors, the organic matter is decontaminated by microorganisms in biogas (methane and carbon dioxide) and compost in the absence of oxygen. Biogas is an additional source of energy that can eventually replace or supplement fossil fuel sources. Thus, it has an indirect positive effect on the reduction of greenhouse gases $[1,2]$. This complex process can become very unstable in open loop. Without the order, the production of this biogas is quickly extinguished. There is need to be implemented strategies for functional control of the biological system carrying out the process of anaerobic digestion [3]. Many mathematical models of these processes in continuously stirred tank reactor (CSTR) are presented by nonlinear ordinary differential equations with a great number of coefficients that are hard to be estimated are known. In these models, a limited number of variables characterizing the process are measurable online. Quite often one obtains only local solutions and it is impossible to validate the model in a large domain of experimental conditions [4]. The five orders AD model is based on the three-stage biochemical scheme and may be accepted as a reasonable compromise between the real process complexity and the realistic basis for theoretical control algorithm design [4].
The operational objective is producing a demanded methane gas flow. Control is important strategy for achieving a better process stability and higher conversion efficiencies in anaerobic digesters. Various control algorithms have been developed to control this complex and strongly nonlinear process as it was mentioned in [5]. Unfortunately these algorithms are often tested on biogas described by a second-order mode. The objective of this paper is to propose a robust control algorithm for a biogas described by a fifth-order model that can be experimentally implemented.

An extended Luenberger observer predicts parameters that require expensive equipment or cannot be measured directly. The realization of software sensors is a preferable method of continuous monitoring of some key process variables and of using this information to make decisions regarding the digester loading, either through automatic organic loading rate systems or advice to operators. This has economic sense in terms of reduced capital costs and improved biogas output.

Main contributions of this paper, in summary, are as follows:

1) A synergetic design procedure is employed to derive the control output of the five-order nonlinear dynamic system, then that it can achieve a high robustness, fast convergence, low steady state error, and globally uniformly asymptotic stability.

2) Proposed observer, used by the command, provides high state estimation. The proposed approach does not introduce any additional complexity. 
3) In comparison with other Pilot-scale Biogas Plant proposed (state estimation algorithms) such as where $S_{2}$ sensor is used [6]. The proposed algorithm is cheaper and provides a good performance by simulation and without focusing on the uncertainty issues of modelling.

The paper begins with a description of the process presented in section 2 with a mathematical model of biogas production by anaerobic digestion. In section 3, the theory and principle of synergetic control are briefly outlined. Observability and recovery are then developed in section 4. The extended Luenberger observer already well known in the literature is recalled in section 5. The simulation results are presented and discussed in the following section. Finally, the reported work is finished with a conclusion.

\section{MATHEMATICAL MODELING OF THE ANAEROBIC DIGESTION}

Different mathematical models of AD exist in the specialized literature: One-stage, Three-stage and Fivestage model. The model for the description of the $\mathrm{AD}$ process used in one stage study were controlled and observed in reference [5]. However, such models don't describe very well the anaerobic digestion behavior. The conversion of volatile fatty acids into biogas is considered limiting. This is the first and the most simplified model for description of the AD processes describing the methanogen step. According to one stage model, a digester is expected to fail whenever, for some reason, the fatty acid concentration is increased. This causes a drop in the ph. and a rise in the acetic acid concentration. This in turn causes a drop in the growth rate of the methanogenic population, until they are washed out, if the situation is prolonged.

Hill and Barth [7] involve an acidogenic step forms acetate from glucose and inhibited by acetic acid. In this reference, the authors considered hydrolysis (enzymatic degradation of insoluble organics to soluble organics), acidogenesis (transformation of the soluble organics to acetate) and methanogenesis (transformation of the acetate to methane) to develop a model of animal waste as the tri-phasic model. Three steps of mechanization are considered. The first is the hydrolysis and liquefaction of insoluble organic matter by extracellular enzymes. The second step describes the acid production phase (acidogen) and the last step illustrates the methanogen phase.

Five-stage model describes the biogas process very well but it is very complex and very difficult to exploit in the control.
The model used in this study is a Three-stage model used in $[4,6]$ devoted to the modeling of anaerobic digestion obtained by fermentation. The dynamics of the anaerobic digestion system represented is illustrated by the following first order differential equations:

$$
\begin{aligned}
& \left\{\begin{array}{l}
\frac{d S_{0}}{d t}=-b X_{1} S_{0}+D Y_{P} S_{i n}-D S_{0} \\
\frac{d X_{1}}{d t}=\left(\mu_{1}-D\right) X_{1} \\
\frac{d S_{1}}{d t}=-k_{1} \mu_{1} X_{1}+b X_{1} S_{0}-D S_{1} \\
\frac{d X_{2}}{d t}=\left(\mu_{2}-D\right) X_{2} \\
\frac{d S_{2}}{d t}=-k_{2} \mu_{2} X_{2}+Y_{b} \mu_{1} X_{1}-D S_{2}
\end{array}\right. \\
& Q=Y_{g} \mu_{2} X_{2} \\
& \mu_{i}=\mu_{i \max } \frac{S_{i}}{S_{i}+k_{S_{i}}} \quad \mathrm{i}=1,2
\end{aligned}
$$

The variables in this model are:

$X_{1}$ and $X_{2}$ are respectively the concentration of the acidogenic bacterial population and the concentration of the methanogenic bacterial population;

$S_{0}, S_{1}, S_{2}$ and $S_{i n}$ are respectively the substrate concentration of complex organic materials in the bioreactor, the concentration of the simple organic material substrate in the bioreactor, the concentration of volatile fatty acid (acetate) substrate in the bioreactor and the concentration of inlet substrate;

$k_{i}(1,2)$ are yield coefficients. $Y_{p}, b, Y_{b}, Y_{g}$ are coefficients. $\mu_{1 \max }, \mu_{2 \max }$ are the maximum specific growth rate $\left(d a y^{-1}\right) \cdot k_{S_{1}}, k_{S_{2}}$ are saturation parameter $(\mathrm{g} / \mathrm{l}) . Q$ the measured biogas flow rate is expressed by $l /$ day. $D\left(d a y^{-1}\right)$ is the dilution rate (the control input).

The relation 3 presents the specific growth rate of the acidogenic bacteria $\mu_{1}\left(d a y^{-1}\right)$ and the specific growth rate of the methanogenic bacteria $\mu_{2}\left(d a y^{-1}\right)$, both of Monod Type.

The state vector is $x=\left(\begin{array}{l}S_{0} \\ X_{1} \\ S_{1} \\ X_{2} \\ S_{2}\end{array}\right)$, the input is $D$ and the output is $Q$. 


\section{PRINCIPLE AND DEVELOPMENT OF SYNERGETIC CONTROL}

The synergetic control theory was first introduced in a general way by Russian researchers in the last years [8]. The principle of synergetic control has been largely explained in the paper [5], one starts with the choice of a macro-variable which is a linear combination, in general, of state variables, taking into account loads the control constraints as well as the specifications:

$$
\Psi=\psi(x, t)=0
$$

$\Psi$ is the macro-variable and $\psi(\boldsymbol{x}, \boldsymbol{t})$ is a function of the state variables and the time chosen by the designer. The synergetic command forces the system to evolve on the domain chosen by the user. The macro-variable is forced to evolve in a desired manner according to a chosen constraint indicated as an example by the following first-order differential equation:

$$
T \dot{\psi}+\psi=0 \quad T>0
$$

$\mathrm{T}$ indicates the convergence speed of the closed- loop system. For such a differential equation of the first order, more $\mathrm{T}$ is small, plus the convergence is fast.

Obviously since the solution of this differential equation is given by $\psi=e^{-\bar{T}}$.

The command can thus be obtained:

$$
u=g(x, \psi(x, t), T, t)
$$

The command depends on the state variables of the system, the macro-variable and the parameter $T$.

This approach does not require the linearization of the model and explicitly uses a nonlinear model for the synthesis of the command.

By the appropriate choice of macro-variables, the designer can obtain the following interesting features for the final system [9]:

- Overall stability

- Insensitivity to the parameters

- Noise suppression.

For the process considered in this paper, the macro-variable was defined by:

$$
\Psi=\mathrm{e}=\mathrm{Q}_{\mathrm{d}}-\mathrm{Q}
$$

The constraint defined by the equation gives:

$$
\begin{aligned}
& T\left(\dot{Q}_{d}-\dot{Q}\right)+\Psi=0 \\
& \dot{Q}=Y_{g}\left(\dot{\mu} X_{2}+\mu \dot{X}_{2}\right) \\
& \dot{Q}=Y_{g} X_{2}\left(\frac{\mu_{2 \max } \dot{S}_{2}\left(k_{S_{2}}+S_{2}\right)-\mu_{2 \max } \dot{S}_{2} S_{2}}{\left(k_{S_{2}}+S_{2}\right)^{2}}+\mu_{2}\left(\mu_{2}-D\right)\right)
\end{aligned}
$$

Replacing $\dot{S}_{2}$ with its value, one obtains

$$
\dot{Q}=Y_{g} X_{2}\left(\frac{\mu_{2 \max }\left(-Y_{2} \mu_{2} X_{2}+Y_{b} \mu_{1} X_{1}-D S_{2}\right)}{\left(k_{S_{2}}+S_{2}\right)^{2}} k_{S_{2}}+\mu_{2}\left(\mu_{2}-D\right)\right)
$$

By replacing in equation (8), one gets

$$
-T Y_{g} X_{2}\left(\frac{\mu_{2 \max }\left(-Y_{2} \mu_{2} X_{2}+Y_{b} \mu_{1} X_{1}-D S_{2}\right)}{\left(k_{S_{2}}+S_{2}\right)^{2}} k_{S_{2}}+\mu_{2}\left(\mu_{2}-D\right)\right)+\Psi=0
$$

After development, one gets

$$
D=\frac{-\frac{\Psi}{T Y_{g} X_{2}}+\frac{\mu_{m 2}\left(-Y_{2} \mu_{2} X_{2}+Y_{b} \mu_{1} X_{1}\right)}{\left(k_{S 2}+S_{2}\right)^{2}} k_{S 2}+\mu_{2}^{2}}{\frac{\mu_{2}}{\left(k_{S 2}+S_{2}\right)} k_{S 2}+\mu_{2}}
$$


It is to be noted that biomass concentration $X_{2}$ is never null.

The command $D$ depends on $X_{2}, X_{1}, \mu_{1}, \mu_{2}$, $S_{2}$ which are not measured. $\mu_{1}$ depends on $S_{1}$ which in turn depends on $S_{0}$. Observers must be proposed to estimate the state variables. Before proposing observers, observability study will be approached.

\section{OBSERVABILITY AND RECOVERY}

The analysis of nonlinear systems observability in view of control is an important field of research to implement observers in the system. In case of linear systems, observability notions are well-known and independent of any state space region where the system is working. These can be global, partial or of an eigenvalue and so and so on. Then, system properties are global. In case of nonlinear system, observability notions are more difficult and linked to operating point [10]. Generally, system properties are locally determined.

Let's consider this continuous nonlinear system:

$$
\left\{\begin{array}{l}
\dot{x}(\mathrm{t})=f(x(\mathrm{t}), u(\mathrm{t}), \mathrm{t}) \\
y(\mathrm{t})=h(x(\mathrm{t}), u(\mathrm{t}), \mathrm{t})
\end{array}\right.
$$

where $\mathrm{x} \in \mathfrak{R}^{\mathrm{n}}$ is the state vector, $\mathrm{u} \in \mathfrak{R}^{\mathrm{m}}$ the input vector, $y \in \mathfrak{R}^{p}$ the output vector and $f, g$ and $h$ are function of proper dimension. one must build the observability matrix and requires that the rank of this matrix is full $(=\mathrm{n}$, the dimension the state of the system).

Nonlinear observability can be done by making an observabitlity matrix using lee algebra and simply look for the full rank of the matrix as is the case in linear systems. The nonlinear system given by (14) is generically observable except for particular values of $x$ and $u$ if and only if:

$$
\operatorname{rank}(O)=\operatorname{rank}\left\{\begin{array}{c}
\frac{\partial h}{\partial x} \\
\frac{\partial L_{f} h}{\partial x} \\
\vdots \\
\frac{\partial L_{f}^{n-1} h}{\partial x}
\end{array}\right\}=n
$$

with $L_{f} h$ the Lie derivative of $\mathrm{h}$ along the vector field $f$. It is a $p$-dimensional vector.
This theory can be applied in our process:

$$
\begin{aligned}
& f(x(t), u(t), t)=\left(\begin{array}{l}
-b X_{1} S_{0}+D Y_{P} S_{\text {in }}-D S_{0} \\
\left(\mu_{1}-D\right) X_{1} \\
-k_{1} \mu_{1} X_{1}+b X_{1} S_{0}-D S_{1} \\
\left(\mu_{2}-D\right) X_{2} \\
-k_{2} \mu_{2} X_{2}+Y_{b} \mu_{1} X_{1}-D S_{2}
\end{array}\right) \\
& h(x(t), u(t), t)=\left(\begin{array}{lllll}
0 & 0 & 0 & Y_{g} \mu_{2} & 0
\end{array}\right)\left(\begin{array}{l}
S_{0} \\
X_{1} \\
S_{1} \\
X_{2} \\
S_{2}
\end{array}\right)
\end{aligned}
$$

A Matlab program was written to study the observability matrix and to test the observability index. The results show that biogas sensor (Q) guarantees the observability of the system.

The $\operatorname{rank}(\mathrm{O})$ represents the observability index of the sensor Q. This index is equal 5.

To test the set of state variables that can be estimated by this sensor, we move on to the concept of observability recovery. the objective of the notion of recovery is to identify the state variables that can be observed by the sensor considered ( $\mathrm{Q}$ in our case) Let $\mathrm{Q}$ be the process sensors; by definition, the state space $\mathrm{E}(\mathrm{x})$ is observable by the available sensor system if the sensor participates in the coverage of a certain sub-space of the state space. The system can be observable but without being able to observe all the state variables. For example, the sensor used may observe some state variables but not others.

The functional $\mathrm{E}(\mathrm{x})$ is observable by the sensor $Q$ if and only if

$$
\operatorname{rank}\left[\frac{\partial E(x)}{\partial x}\right]=\operatorname{rank}(\mathrm{O})
$$

A Matlab program was written to calculate the observability recovery. $E(x)$ will take 5 different values to test the recovery of 5 state variables:

$$
\begin{aligned}
& E(x)=\left(\begin{array}{lllll}
S_{0} & 0 & 0 & 0 & 0
\end{array}\right) \\
& E(x)=\left(\begin{array}{lllll}
0 & X_{1} & 0 & 0 & 0
\end{array}\right) \\
& E(x)=\left(\begin{array}{lllll}
0 & 0 & S_{1} & 0 & 0
\end{array}\right) \\
& E(x)=\left(\begin{array}{lllll}
0 & 0 & 0 & X_{2} & 0
\end{array}\right) \\
& E(x)=\left(\begin{array}{lllll}
0 & 0 & 0 & 0 & S_{2}
\end{array}\right)
\end{aligned}
$$

The observability recovery Matlab programs indicates that the different cell concentration $\left(S_{0}\right.$, 
$S_{1}, S_{2}, X_{1}$ and $X_{2}$ ) can be estimated by the sensor

$\mathrm{Q}$ because the result obtained for the equation (16) were 5 whatever is $E(x)$.

\section{EXTENDED LUENBERGER OBSERVER DESIGN FOR IMMEASURABLE STATE VARIABLES OF AD PROCESSES}

Observers (software sensors) are important for gaining of the bacteria and substrate participating in AD processes. An extended Luenberger observer was used for AD Processes in [11] and an adaptative extended luenberger observer for nonlinear system in [12].

The extended Luenberger observer principle is directly inspired by the linear case. The extended Luenberger observer requires knowledge of the model, including system inputs.

Consider the system given by equation (14), the extended Luenberger Observer Synthesis gives rise to the following structure:

$$
\dot{\bar{x}}(t)=f(\dot{\bar{x}}(t), u(t), t) \text { with } \hat{x}\left(t_{0}\right)=\hat{x}_{0}
$$

For the AD process described by equation (1), the states estimation gives equation system (17):

$$
\left\{\begin{array}{l}
\frac{d \hat{S}_{0}}{d t}=-b \hat{X}_{1} \hat{S}_{0}+D Y_{P} S_{i n}-D \hat{S}_{0}+L_{S_{0}} \tilde{Q} \\
\frac{d \hat{X}_{1}}{d t}=\left(\hat{\mu}_{1}-D\right) \hat{X}_{1}+L_{X_{1}} \tilde{Q} \\
\frac{d \hat{S}_{1}}{d t}=-k_{1} \hat{\mu}_{1} \hat{X}_{1}+b \hat{X}_{1} \hat{S}_{0}-D \hat{S}_{1}+L_{S_{1}} \tilde{Q} \\
\frac{d \hat{X}_{2}}{d t}=\left(\hat{\mu}_{2}-D\right) \hat{X}_{2}+L_{X_{2}} \tilde{Q} \\
\frac{d \hat{S}_{2}}{d t}=-Y_{2} \hat{\mu}_{2} \hat{X}_{2}+Y_{b} \hat{\mu}_{1} \hat{X}_{1}-D \hat{S}_{2}+L_{S_{2}} \tilde{Q}
\end{array}\right.
$$

where $\tilde{Q}=Q-\hat{Q}$

Once $S_{1}$ and $S_{2}$ are estimated, the values of $\mu_{1}$ and $\mu_{2}$ can be evaluated using equation (3). Then recalculate $\mathrm{D}$ from all these values. $\mathrm{D}$ expressed by equation (13) can be obtained by the observed variables as shown in equation (18):

$$
D=\frac{-\frac{\Psi}{T Y_{g} \hat{X}_{2}}+\frac{\mu_{m 2}\left(-Y_{2} \hat{\mu}_{2} \hat{X}_{2}+Y_{b} \hat{\mu}_{1} \hat{X}_{1}\right)}{\left(k_{S 2}+\hat{S}_{2}\right)^{2}} k_{S 2}+\hat{\mu}_{2}^{2}}{\frac{\hat{\mu}_{2}}{\left(k_{S 2}+\hat{S}_{2}\right)} k_{S 2}+\hat{\mu}_{2}}
$$

In this way, all measurable and non-measurable variables are shown in real time on a computer through the only measurement of Q as in the paper [6]. But in this reference, 2 measures were used biogas flow rate $Q$ and concentration of volatile fatty acid (acetate) substrate $S_{2}$. This principle, used in $\mathrm{AD}$, can detect real-time failures in the process.

\section{SIMULATION AND RESULTS}

The parameters used in the simulation are in $[4,6]$. Those parameters are used in $100 \mathrm{~L}$ bioreactor used to develop $\mathrm{AD}$ technologies based on standard feedstock such as diluted and suspended swine or cattle manure or chicken litter from agricultural farms, separately or in co-digestion with wasted fruits and vegetables from stock markets, grinded straw, restaurant wastes, activated sludge from the Waste Water Treatment Plants, etc. at mesophilic temperature $\left(34^{\circ} \mathrm{C}\right)$. The scheme of this pilot plant with a system for monitoring and control can be integrated in an experimental.

The numerical implementation was carried out by using the Matlab-Simulink software. The model parameters used during the simulation runs are:

$$
\begin{aligned}
& S_{0}(0)=10 \mathrm{~g} / \mathrm{l}, \quad X_{1}(0)=0.36 \mathrm{~g} / \mathrm{l}, \\
& S_{1}(0)=S_{2}(0)=0.18 \mathrm{~g} / \mathrm{l}, \\
& X_{2}(0)=15.66 \mathrm{~g} / \mathrm{l} .
\end{aligned}
$$

The observer gains are chosen as:

$$
L_{S_{0}}=0.5, L_{S_{1}}=2, L_{S_{2}}=0.2, L_{X_{1}}=2, L_{X_{2}}=2 \text {. }
$$

The initial conditions for the observer are

$$
\begin{aligned}
& \hat{S}_{0}(0)=10.5 \mathrm{~g} / \mathrm{l}, \hat{X}_{1}(0)=0.86 \mathrm{~g} / \mathrm{l}, \\
& \hat{S}_{1}(0)=\hat{S}_{2}(0)=0.68 \mathrm{~g} / l, \hat{X}_{2}(0)=16.16 \mathrm{~g} / \mathrm{l} .
\end{aligned}
$$

Note that there is a significant error between the initial conditions of the real states and the estimated states, which was induced in order to test the observer performance.

Starting with $\tilde{Q}(0) \neq 0$ as shown in Fig. 2 good tracking occurs under the proposed observer based synergetic controller.

Fig. 2 proves the robustness of the command presented in this paper, synergetic control, The biogas flow rate $(\mathrm{Q})$ tracking with desired biogas flow rate $\left(Q_{d}\right)$ shows the good performances of the system. In the zoomed curve, there is a shift between estimated biogas flow rate $\left(Q_{e s t}\right)$ and desired biogas flow rate $\left(Q_{d}\right)$ from the observer and not the command. 


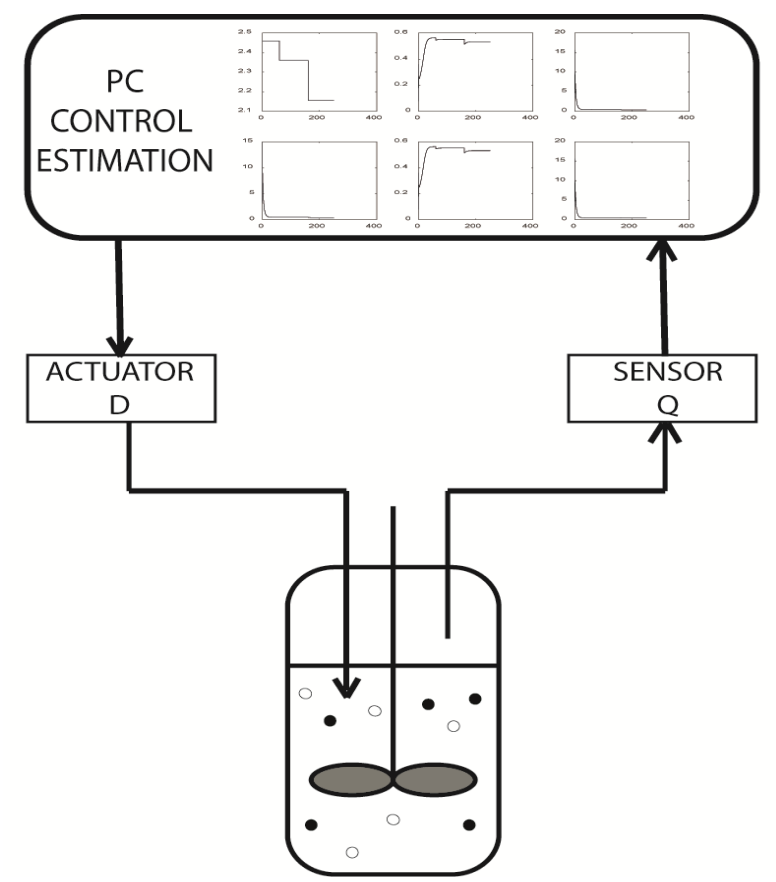

Fig. 1. Scheme for monitoring, estimation and control of AD process

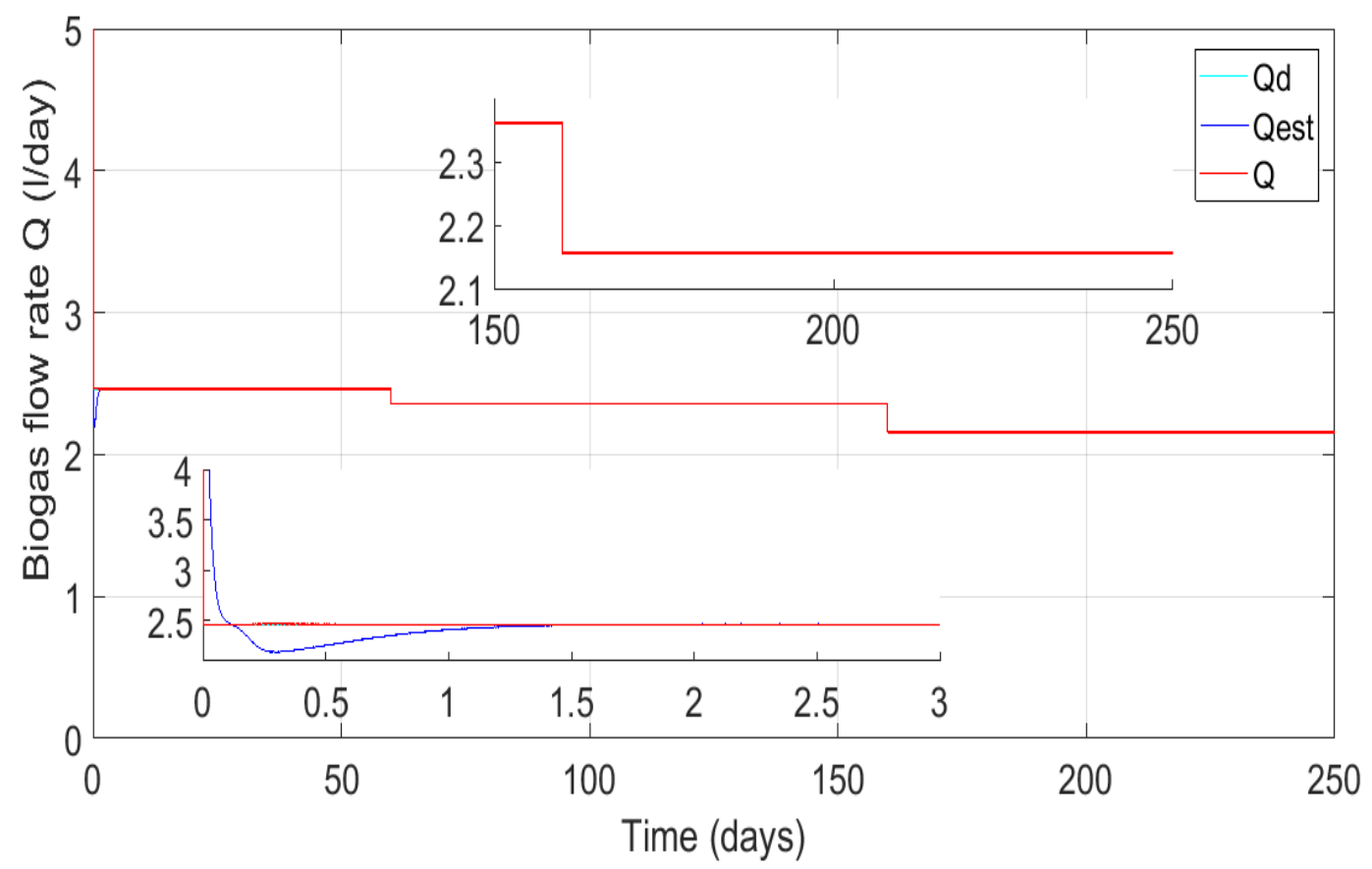

Fig. 2. Time evolution of biogas flow rate under synergetic control for $\mathrm{T}=0.0001$ 


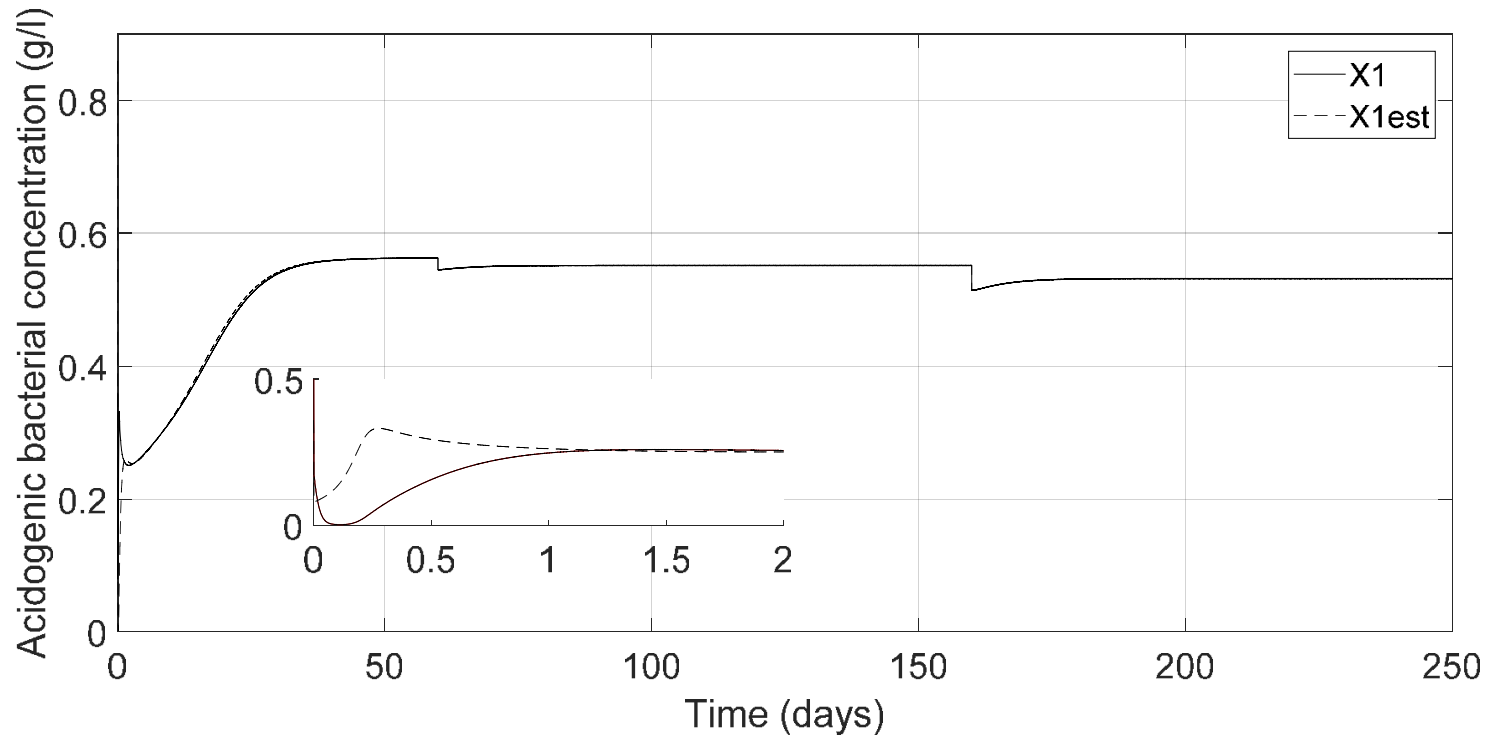

Fig. 3. Real acidogenic bacterial concentration evolution $X_{1}$ and its estimation $\hat{X}_{1}$

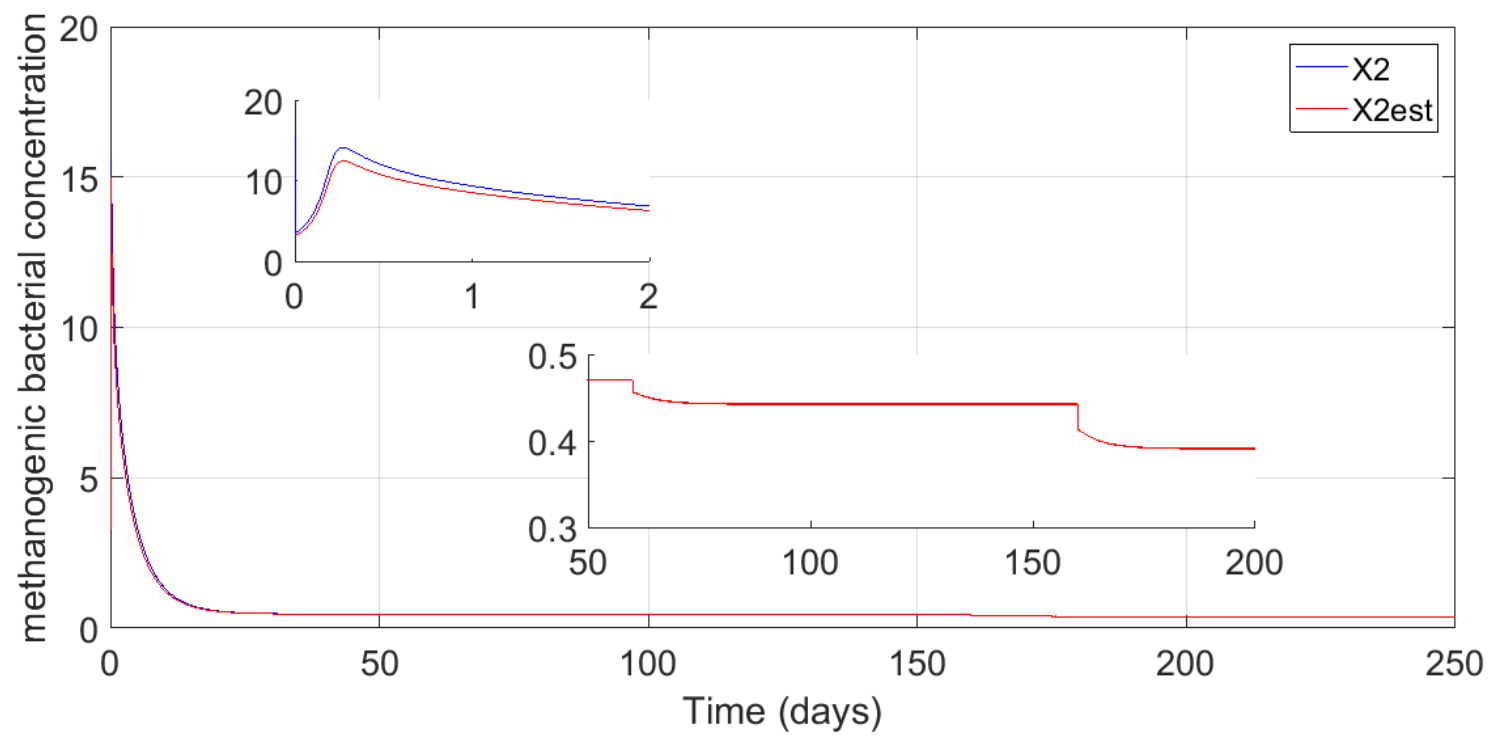

Fig. 4. Real methanogenic bacterial concentration evolution $X_{2}$ and its estimation $\hat{X}_{2}$

Figures 3 and 4 the convergence of estimated acidogenic bacterial concentration $\left(\hat{X}_{1}\right)$ and estimated metanogenic bacterial concentration $\left(\hat{X}_{2}\right)$ is in finite time.

Figures 5, 6 and 7 show the estimation error for states $\quad \tilde{S}_{0} \quad \tilde{S}_{1}$ and $\tilde{S}_{2}$. Regarding convergence, we note that our extended Luenberger observer has excellent convergences for the substrates those are obtained in finite time.
$\tilde{S}_{0}$ express the difference between substrate concentration of complex organic materials in the bioreactor $S_{0}$ and its estimation $\hat{S}_{0}$.

$\tilde{S}_{1} \quad$ express the difference between the concentration of the simple organic material substrate in the bioreactor $S_{1}$ and its estimation $\hat{S}_{1}$.

$\tilde{S}_{2}$ express the difference between the concentration of volatile fatty acid substrate in the bioreactor $S_{2}$ and its estimation $\hat{S}_{2}$. 
As substrate concentrations are estimated, the the specific growth rate of the methanogenic bacteria and specific growth rate of the acidogenic bacteria $\mu_{1}$ and $\mu_{2}$ can be easily calculated using the relationships (3).

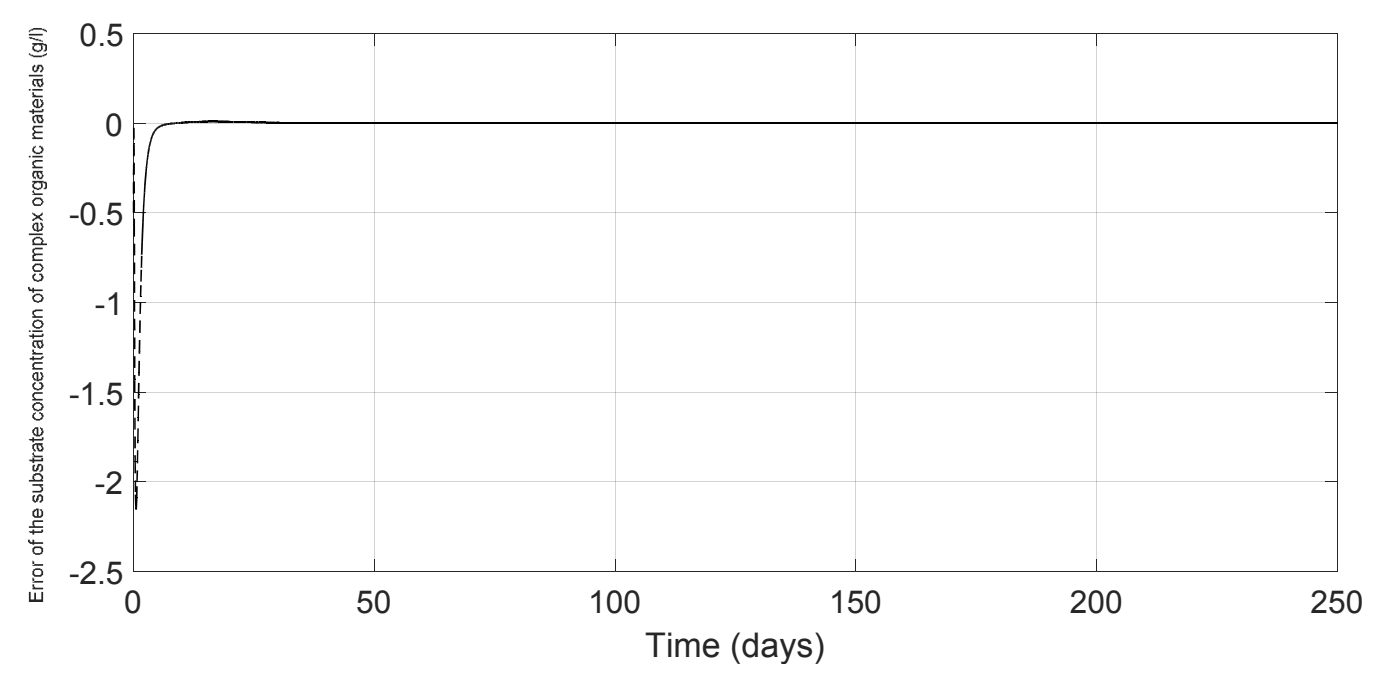

Fig. 5. Error of the substrate concentration of complex organic materials in the bioreactor evolution $\tilde{S}_{0}$

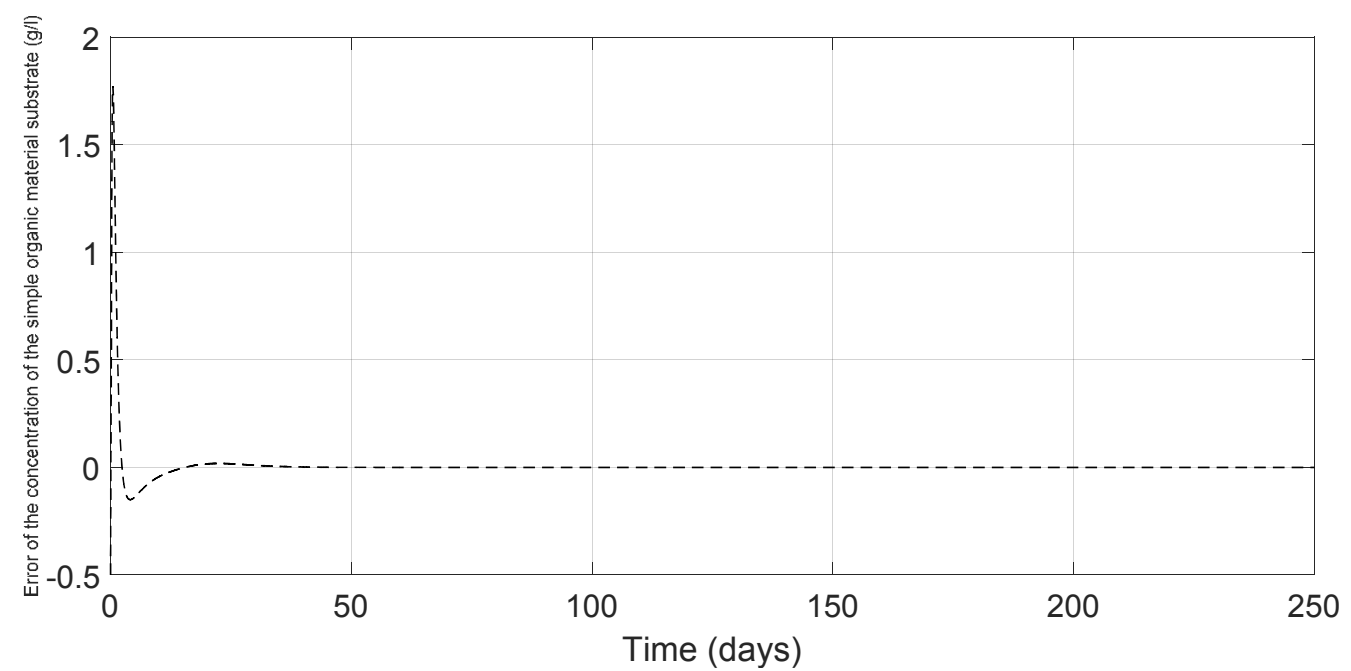

Fig. 6. Error of the concentration of the simple organic material substrate evolution in the bioreactor $\tilde{S}_{1}$

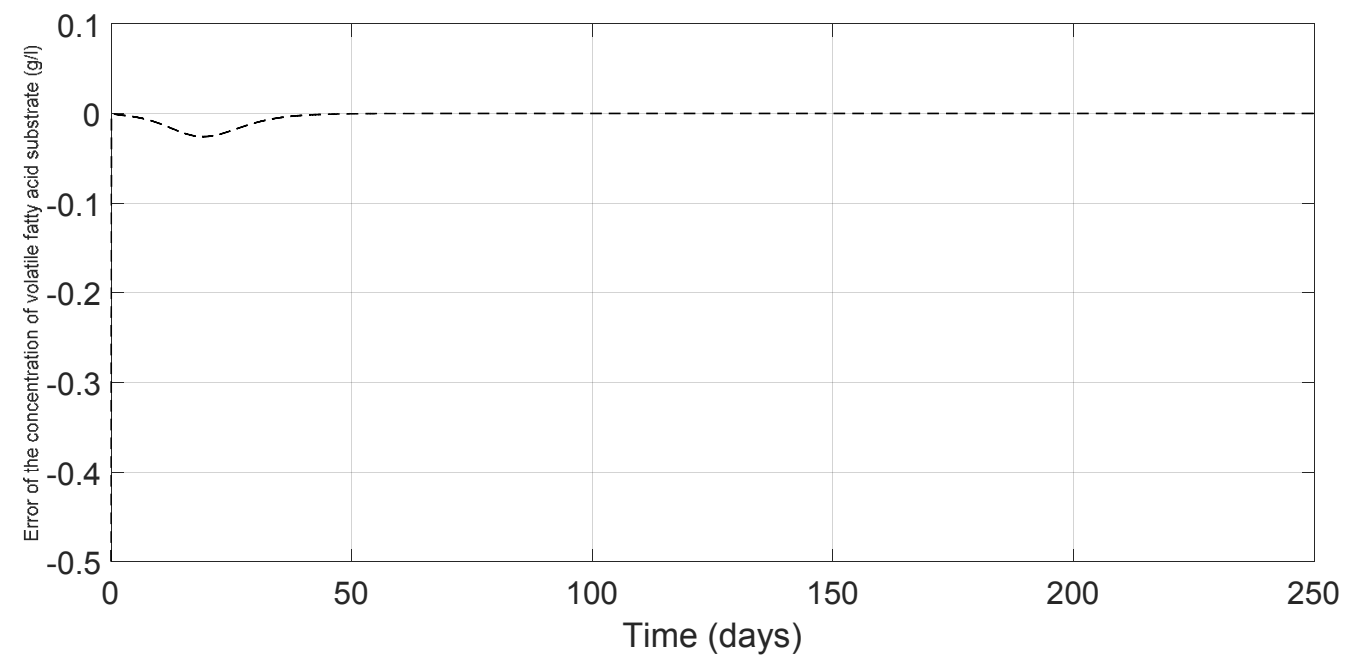

Fig. 7. Error of the concentration of volatile fatty acid substrate evolution in the bioreactor $\tilde{S}_{2}$ 


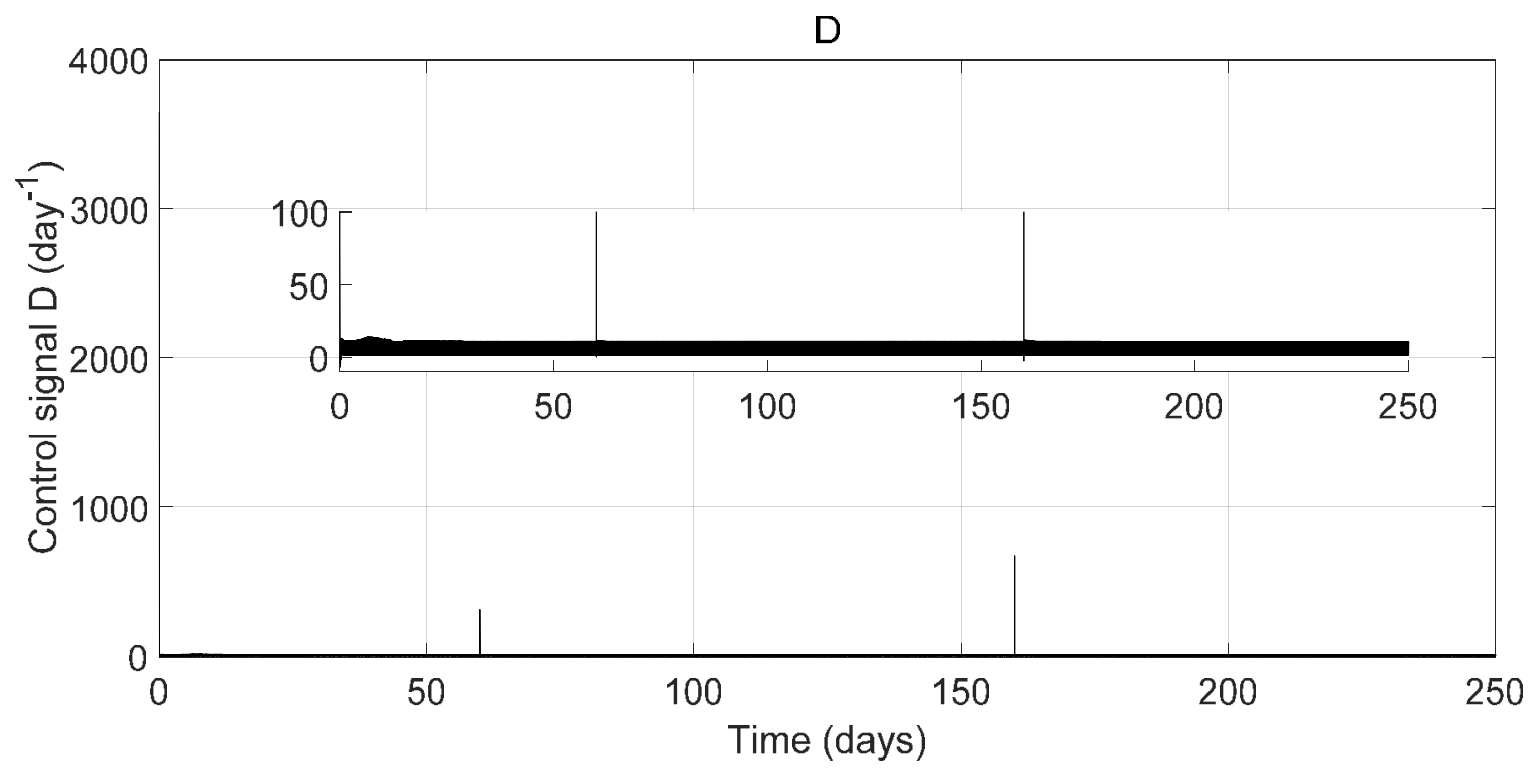

Fig. 8. Control signal evolution under synergetic control for $\mathrm{T}=0.0001$

At Fig. 8, the control signal is always positive and reaches at 60 day and 160 day which corresponds to the jumps in the desired biogas flow rate.

\section{CONCLUSION}

Biogas has recently received a lot of attention because of its potential in preserving the environment and as a cheap source of renewable energy. Nevertheless biogas production requires robust control techniques mainly for its strongly nonlinear model; as such a synergetic controller has been proposed and validated. The proposed software sensors for $\mathrm{AD}$ unmeasurable variables are model dependent. Software sensors for estimation of acidogenic and methanogenic bacterial concentrations and specific growth rates have been designed on the basis of the three-stage AD model. They are much simple and easily realizable, measuring only biogas flow rate. The performances of the observer depend on the precise values of model parameters.

The control scheme combines an output feedback control with Luenberger observer, which allowed the estimation of the uncertain terms associated to the controlled states. It was shown through numerical simulations that the proposed control scheme was capable to maintain the desired set-points practical stability criteria. The experimental implementation of the proposed control scheme can be developed.

\section{REFERENCES}

1. Viesturs U., I. Simeonov, T. Pencheva, J. Vanags, M. Petrov, Y. Pavlov, O. Roeva, T. Ilkova, M. Vishkins, I. Hristozov, Contemporary approaches to modelling, optimization and control of biotechnological processes), Stoyan Tzonkov, Sofia, 2010, $241 \mathrm{p}$.

2. Baldé Y.M., C.Kanté, S. Diop, S. Tebbani, An experimental study for the characterization of biogas production from cow dung and droppings, Ecological Engineering and Environment Protection, $\mathrm{n}^{\circ} 1,2019,54-61$.

3. Dinova N., M. Belouhova, I. Schneider, Y.Topalova, Functional control of the technologies for biogas production, Ecological Engineering and Environment Protection, ${ }^{\circ} 1$, 2016, 52-64.

4. Wang H.P., Tian Y., Kalchev B., I. Simeonov, Pilot-scale biogas plant: description, modelling and composed recursive control), CEAI, vol.15, n02, 2013, 38-45.

5. Bouchareb H., S. Semcheddine, M. N. Harmas, K.N. M'sirdi, A. Naamane, Virtual sensors to drive anaerobic digestion under a synergetic controller, Energy, Vol 12, 3, 430, 2019.

6. Simeonov I., S. Diop et al., Design of software sensors for unmeasurable variables of anaerobic digestion processes. new trends in microbiology, 65th Anniversary of the Stephan Angeloff Institute of Microbiology, the Stephan Angeloff Institute of Microbiology, Bulgarian Academy of Sciences, Bulgaria, 2012, 307-311. 
7. Hill D.T, C.L.Barth, A dynamic model for simulation of animal waste digestion, Water Pollution Control Federation, Vol.49, 10, 1977, 2129-2143.

8. Wang Q., J. Feng, T. Li, Analysis of the synergetic control based on variable structure and application of power electronics, International conference on information engineering and computer science, Wuhan, China, 2009, 9-12.

9. Li D., K. Proddutur., E. Santi, A. Monti, Synergetic control of a boost converter: Theory and experimental verification, Proc. IEEE, Columbia, SC, USA, 2002.197-200.

10. Hermann R., A. J. Krener, Nonlinear controllability and observability, IEEE Transactions on Automatic Control, Vol. AC-22, No. 5, October 1977, 728-740.

11. Méndez-Acosta H. O., B. Palacios-Ruiz, V. Alcaraz-González, V. González-Álvarez, J. P. García-Sandoval, A robust control scheme to improve the stability of anaerobic digestion processes, Journal of Process Control, 20, 2010, 375-383.

12. Umut Gen A., S. Auddy, D. Erdogmus, S.P. Das, J. C. Principe, Adaptive extended luenberger observer in state feedback control of nonlinear dynamical systems,

https://www.researchgate.net/publication/228957657

\section{Samia Semcheddine, Hanane Bouchareb}

LEPCI laboratory, faculty of technology,

Ferhat Abbas University, Setif1, Algeria

E-mails: samia.semchedine@univ-setif.dz, hananebouchareb@yahoo.fr 